\begin{tabular}{|c|c|c|}
\hline & Int.J.Curr.Microbiol.App.Sci (2020) 9(1): 152-159 & \\
\hline & \multirow[t]{2}{*}{$\begin{array}{l}\text { International Journal of Current Microbiology and Applied Sciences } \\
\text { ISSN: 2319-7706 Volume } 9 \text { Number } \mathbf{1}(\mathbf{2 0 2 0 )} \\
\text { Journal homepage: } \underline{\text { http://www.ijcmas.com }}\end{array}$} & 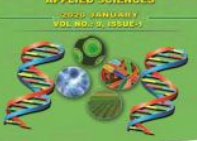 \\
\hline $\begin{array}{l}\text { EXCELLENT } \\
\text { PUBLISHERS }\end{array}$ & & \\
\hline
\end{tabular}

Original Research Article

https://doi.org/10.20546/ijcmas.2020.901.017

\title{
Different Types of Soil Acidity as Influenced by Nitrogen Application and Soil Types in Dhamtari Block of Chhattisgarh, India
}

\author{
Hareesh Kumar*, S. S. Sengar, Bharat Desmukh and R. N. Singh \\ Department of Soil Science and Agricultural Chemistry Indira Gandhi Agricultural \\ University, Raipur-492 012, C.G, India \\ *Corresponding author
}

\section{A B S T R A C T}

\section{Keywords}

Soil acidity, soil types, forms of acidity, Dhamtari, Chhattisgarh

Article Info

Accepted:

15 December 2019

Available Online:

20 January 2020
Soil acidification is caused by a number of factors including acidic precipitation and the deposition from the atmosphere of acidifying gases or particles, such as sulphur dioxide, ammonia and nitric acid. The most important causes of soil acidification on agricultural land, however, are the application of ammonium-based fertilizers and urea, elemental $\mathrm{S}$ fertilizer and the growth of legumes. Soil samples were collected over a period of 2017-2018 from the entire block, to study physico-chemical parameters of three seasons. A comparative study has been carried out in order to study the extent of pollution caused due to application of chemical fertilizers to soil and water systems. Relative order for all forms of acidity was Inceptisol >Alfisols $>$ Vertisol. On average contribution of soil acidity due to TPA in Inceptisol, Alfisol and Vertisol was found maximum $35.5 \%, 35.6 \%$ and $32.3 \%$ at $0-30 \mathrm{~cm}$ soil depth, 36.0 $\%, 34.3 \%$ and $33.0 \%$ at $30-60 \mathrm{~cm}$ soil depth and $35.5 \%, 34.6 \%$ and $33.0 \%$ at 60 $90 \mathrm{~cm}$ soil depth, respectively, while, the minimum contribution in soil acidity was due to exchangeable acidity i.e. $9.2,8.5$ and $9.2 \%$ at $0-30 \mathrm{~cm}$ soil depth, $7.5 \%, 6.5 \%$ and $7.5 \%$ at $30-60 \mathrm{~cm}$ depth and 5.6, 4.8 and $5.5 \%$ at $60-90 \mathrm{~cm}$ soil depth, respectively. The different forms of soil acidity in Dhamtri block of Chhattisgarh in the following order TPA $>\mathrm{PDA}>\mathrm{Al}^{3+}>\mathrm{TA}>\mathrm{EA}>\mathrm{E}-\mathrm{Al} 3+>$ exchangeable acidity.

\section{Introduction}

Some soils are acidic because of the composition of the parent material (rocks) from which they were formed. Other soils become acid by a number of processes. Cropping and use of nitrogen fertilizers are two main sources of soil acidity while another contributor is rainfall. The net result is that hydrogen, aluminum, and iron (acidic cations) replace calcium, magnesium, potassium, and sodium (basic cations) on the soil cation exchange complex. Soil acidity is an important agricultural problem leading to severe toxicity of iron, aluminium and manganese in many crops, coupled with deficiency of phosphorus and low microbial activity that led to poor yield of crops (Reza $e t$ 
al., 2012) Exchangeable acidity refers to the sum of the concentrations of hydrogen $(\mathrm{H})$ and aluminium (Al) ions in the soil exchange complex and is inversely related to basic cations of the soil. Correlations of the nonexchangeable $\mathrm{Al}$ and forms of soil acidity were positive and significant, indicating the dynamic equilibrium among different forms of aluminium and their role in soil acidity.

The different forms of aluminium had significant contribution to the forms of soil acidity. Therefore, knowledge on different forms of acidity may provide firsthand information on acid soils for their better management. Thus, an attempt has been made here to characterize the different forms of soil acidity in relation to land uses and to evaluate the influence of soil properties on them as very little information is available for soils of Dhamtri block on this aspect.

\section{Materials and Methods}

Ninety (90) soil samples were collected from 10 replications as 10 random farmers for each combination dose of fertilizer $(\mathrm{F})$ and soil type (T) during pre monsoon, during monsoon and post monsoon season in April, August and November 2017 respectively at random depth 0-30, 30-60 and 60-90 cm. Location of sampling point were determined using a Global Positioning System (GPS) presented in table 3.2. They were carefully packed in polythene bags.

The total potential acidity were measured by the method of Peech et al., (1962). The pH dependent acidity was estimated by the following equation: $\mathrm{pH}$ dependent acidity = Total potential acidity - Exchange acidity. Total soil acidity was measured by shaking the soil with $1 \mathrm{~N} \mathrm{NaOAc}$ for an hour (Kappen, 1934); the Exchangeable Al was determined by the principle of Baruah and Barthakur (1999)

\section{Results and Discussion}

Different types of soil acidity as influenced by $\mathbf{N}$ application and soil types under the study area during pre-monsoon season

The contribution of different types of soil acidity in different types of soil at various depth are presented in table 1 . The maximum (36.8\%) contribution on soil acidity was due to total potential acidity (TPA), While, the minimum $(8.9 \%)$ contribution was from exchangeable acidity (Ex A) in Inceptisol under the treatment $<92 \mathrm{~kg} / \mathrm{ha}-\mathrm{N}$ at $0-30 \mathrm{~cm}$. soil depth and similar trends were observed at $30-60 \mathrm{~cm}$. and $60-90 \mathrm{~cm}$. soil depth in Inceptisol under $<92 \mathrm{~kg} / \mathrm{ha}-\mathrm{N}$. The soil acidity of Inceptisol at all 0 - 30,30 - 60 and $60-90 \mathrm{~cm}$. soil depth under same level of nitrogen fertilization followed the order of soil acidity total potential acidity $>\mathrm{pH}$ dependent acidity $>$ total acidity $>$ aluminum acidity $>$ exchangeable acidity. Whereas, the maximum contribution in soil acidity due to TPA (36.3, 34.5 and $34.8 \%$ ) and the minimum from exchangeable acidity i.e. 6.9, 5.4 and 4.2 on soil acidity were observed in Alfisol at 0-30, 30-60 and $60-90 \mathrm{~cm}$ soil depth, respectively, under $<92 \mathrm{~kg} / \mathrm{ha}-\mathrm{N}$ application. However, the maximum contribution in soil acidity of Vertisol was 32.6, 33.8 and $32.8 \%$ and the minimum was $8.9,6.9$ and $5.6 \%$ as a TPA and Ex. A. at 0-30, 30-60 and $60-90 \mathrm{~cm}$ soil depth respectively, at $<92 \mathrm{~kg} / \mathrm{ha}-\mathrm{N}$. While, the soil acidity followed the order in Vertisol as TPA >PDA>Al. A > TA > exchangeable acidity. The mean data on soil acidity were also decreased with increasing soil depth during pre-monsoon season.

The maximum involvement of TPA on soil acidity of Inceptisol, Alfisol and Vertisol 35.7, 35.9 and $33.6 \%$ at $0-30 \mathrm{~cm}$ soil depth, 35.3, 36.2 and $33.7 \%$ at $30-60 \mathrm{~cm}$ soil depth and $35.8,36.3$ and $34.5 \%$ at $60-90 \mathrm{~cm}$ soil depth, respectively and the minimum involvement of 
exchangeable acidity on soils were recorded $10.9,8.9$ and 9.6 at $0-30 \mathrm{~cm}$ soil depth, 7.8, 6.7 and 7.7 at $30-60 \mathrm{~cm}$ soil depth and 6.6,6.4 and 7.0 at $6-90 \mathrm{~cm}$ soil depth, respectively, under the treatment $>138 \mathrm{~kg} / \mathrm{ha}-\mathrm{N}$ application. Whereas, all the above results showed that the soil acidity increased with increasing dose of nitrogenous fertilizer and soil acidity followed the order as Inceptisol >Alfisol>Vertisol.

Soil acidity contribution in Inceptisol, Alfisol and Vertisol was maximum due to TPA i.e. $35.6,36.1$ and $35.8 \%$ at $0-30 \mathrm{~cm}$ depth, 35.3, 36.3 and $33.2 \%$ at $30-60 \mathrm{~cm}$ soil depth and $35.7,36.6$ and $33.4 \%$ at $60-90 \mathrm{~cm}$ soil depth, respectively, under 92-138 kg/ha-N application, while the minimum soil acidity of Inceptisol, Alfisol and Vertisol was exchangeable acidity i.e. 10.1,8.0 and 7.6 at 0$30 \mathrm{~cm}$ depth, $8.3,6.3$ and 6.8 at $30-60 \mathrm{~cm}$ depth and 7.4, 5.4 and $5.5 \%$ at $60-90 \mathrm{~cm}$ soil depth under $92-138 \mathrm{~kg} / \mathrm{ha}-\mathrm{N}$ application. The mean value of soil acidity was decreased from surface to sub-surface soil and soil acidity was found in the order TPA> PDA>Al.A. >TA> Ex. A. in Inceptisol and Vertisol while, TPA $>$ PDA $>$ TA $>$ Al.A.>Ex.A. in Alfisols. Sarangthem et al., (2017) reported that the exchange acidity, $\mathrm{pH}$ dependent acidity and aluminum acidity of the studied soil samples indicated that exchange acidity value was low as compare to total potential acidity. Data showed that exchange acidity have relatively low contribution towards total acidity. Similar findings were also observed by Sharma et al., (2017), Das et al., (1991) and Kumar et al., (1995).

Different types of soil acidity as influenced by $\mathrm{N}$ application and soil type under the study area during mid-monsoon season

The contribution towards different type of soil acidity on different types of soil at various depth are presented in table 2. The contribution of soil acidity due to TPA in
Inceptisol, Alfisol and Vertisol was found maximum $35.5 \%, 35.6 \%$ and $32.3 \%$ at $0-30$ $\mathrm{cm}$ soil depth, $36.0 \%, 34.3 \%$ and $33.0 \%$ at $30-60 \mathrm{~cm}$ soil depth and $35.5 \%, 34.6 \%$ and $33.0 \%$ at $60-90 \mathrm{~cm}$ soil depth, respectively, while, the minimum contribution in soil acidity was due to exchangeable acidity i.e. 9.2, 8.5 and 9.2 at $0-30 \mathrm{~cm}$ soil depth, $7.5 \%$, $6.5 \%$ and $7.5 \%$ at $30-60 \mathrm{~cm}$ depth and 5.6, 4.8 and $5.5 \%$ at $60-90 \mathrm{~cm}$ soil depth, respectively, under the $<92 \mathrm{~kg} / \mathrm{ha}-\mathrm{N}$ application during mid-monsoon season. The acidity in Inceptisol and Alfisol followed increase of TPA $>$ PDA $>$ TA $>$ Al-A $>$ Ex.A while in Vertisol the soil acidity followed increase order of TPA $>$ PDA $>$ Al. A $>$ TA $>$ Ex. A.

The contribution in soil acidity due to TPA of Inceptisol, Alfisol and Vertisol was higher i. e. $34.0 \%$ increased, $35.6 \%$ and $34.4 \%$ at $0-30$ $\mathrm{cm}$ soil depth, $34.8 \%, 35.6 \%$ and $35.2 \%$ at $30-60 \mathrm{~cm}$ depth of soil and $34.0 \%, 36.6 \%$ and $35.6 \%$ at $60-90 \mathrm{~cm}$ soil depth, respectively.

Whereas, the minimum contribution in acidity was due to exchangeable acidity which was $11.1 \%, 11.0 \%$ and $10.7 \%$ at $0-30 \mathrm{~cm}$ soil depth, $9.7 \%, 7.2 \%$ and $8.3 \%$ at $30-60 \mathrm{~cm}$ soil depth and $7.7 \%, 6.5 \%$ and $7.5 \%$ at 60 $90 \mathrm{~cm}$ soil depth under the application of 92$138 \mathrm{~kg} / \mathrm{ha}-\mathrm{N}$ during mid monsoon season.

The maximum contribution in soil acidity of Inceptisol, Alfisol and Vertisol was observed due to TPA which was $35.2 \%, 35.6 \%$ and $33.8 \%$ at $0-30 \mathrm{~cm}$ soil depth. $34.8 \%, 34.7 \%$ and $34.3 \%$ at $30-60 \mathrm{~cm}$ soil depth and $35.5 \%$, $35.0 \%$ and $34.6 \%$ at $60-90 \mathrm{~cm}$ soil depth as TPA, respectively.

Whereas, the lowest contribution in soil acidity was found as exchangeable acidity which was $14.0 \%, 11.0 \%$ and $10.0 \%$ at $0-30$ cm soil depth, $10.6 \%, 7.1 \%$ and $6.6 \%$ at 30- 
$60 \mathrm{~cm}$ soil depth and $8.9 \%, 5.7 \%$ and $4.2 \%$ at 60 - $90 \mathrm{~cm}$ soil depth, respectively, under the heavy application of nitrogenous fertilizer i.e. $>138 \mathrm{~kg} / \mathrm{ha}-\mathrm{N}$ during mid-monsoon season. The Inceptisol and Vertisol were follwed the soil acidity order of TPA $>$ PDA $>$ TA $>$ Al. A $>$ Ex.A, while the Alfisol followed the soil acidity order of TPA >PDA> Al. A> TA> Ex.A. It has also been observed that the soil acidity was decreased with increasing soil depth and the soil acidity was recorded in order of Inceptisol $>$ Alfisol $>$ Vertisol.

Different types of soil acidity as influenced by $\mathbf{N}$ application and soil types under the study area during post-monsoon season

The contribution of different types of soil acidity on different soils at various depths are presented in table 3 . The contribution of soil acidity in Inceptisol, Alfisol and Vertisol was found maximum as TPA $35.5 \%, 35.9 \%$ and $35.8 \%$ at $0-30 \mathrm{~cm}$ soil depth, $36.1 \%, 34.9 \%$ and $34.2 \%$ at $30-60 \mathrm{~cm}$ soil depth and $34.3 \%$, $35.6 \%$ and $35.8 \%$ at $60-90 \mathrm{~cm}$ soil depth, respectively, while, the minimum was exchangeable acidity i.e $9.0 \%, 9.1 \%$ and 7.7 $\%$ at $0-30 \mathrm{~cm}$ soil depth, $7.0 \%, 7.7 \%$ and 8.1 $\%$ at $30-60 \mathrm{~cm}$ depth and $7.9 \%, 7.4 \%$ and 6.4 $\%$ at $60-90 \mathrm{~cm}$ soil depth, respectively, under the $<92 \mathrm{~kg} / \mathrm{ha}-\mathrm{N}$ application during postmonsoon season. Inceptisol and Alfisol were followed the acidity in order of TPA $>$ PDA> TA $>$ Al-A> Ex.A and Vertisol followed soil acidity in order of TPA $>$ PDA $>$ Al. A $>$ TA $>$ Ex.A. The contribution in soil acidity due to TPA of Inceptisol, Alfisol and Vertisol was higher that was $37.6 \%, 36.8 \%$ and $35.6 \%$ at 0-30 cm soil depth, $36.2 \%, 36.3 \%$ and $34.3 \%$ at 30-60 $\mathrm{cm}$ depth of soil and $35.8 \%, 36.0 \%$ and $35.14 \%$ at $60-90 \mathrm{~cm}$ soil depth, respectively. Whereas, the minimum contribution was exchangeable acidity which $10.2 \%, 10.5 \%$ and $9.5 \%$ at $0-30 \mathrm{~cm}$ soil depth, $7.7 \%, 7.5 \%$ and $6.8 \%$ at $30-60 \mathrm{~cm}$ soil depth and 8.1\%, $6.3 \%$ and $5.5 \%$ at 60 $90 \mathrm{~cm}$ soil depth under the application of 92$138 \mathrm{~kg} / \mathrm{ha}-\mathrm{N}$ during post- monsoon season.

Highest contribution in soil acidity of Inceptisol, Alfisol and Vertisol was observed due to TPA which was $36.3,36.7$ and $35.1 \%$ at $0-30 \mathrm{~cm}$ soil depth. $36.2,37.5$ and $34.5 \%$ at $30-60 \mathrm{~cm}$ soil depth and $36.3,37.5$ and $37.0 \%$ at $60-90 \mathrm{~cm}$ soil depth, respectively. Whereas, the lowest contribution soil acidity was found as exchangeable acidity which was 11.0, 11.0 and $8.9 \%$ at $0-30 \mathrm{~cm}$ soil depth, 9.7, 6.4 and $7.4 \%$ at $30-60 \mathrm{~cm}$ soil depth and 7.8, 7.0 and $5.3 \%$ at $60-90 \mathrm{~cm}$ soil depth, respectively, under the heavy application of nitrogenous fertilizer i.e. > $138 \mathrm{~kg} / \mathrm{ha}-\mathrm{N}$ during postmonsoon season.

The Inceptisol and Vertisol were followed the soil acidity in order of TPA >PDA $>$ TA $>$ Al. A $>$ Ex.A, while the Alfisol followed the soil acidity order of TPA >PDA $>$ Al. A>TA> Ex.A.it has also been observed that the soil acidity was decreased with increasing soil depth however, in different soil types the soil acidity was found in order of Inceptisol $>$ Alfisol > Vertisol.

Mandal et al., (2006) reported that the lower value of total acidity may be due to low contents of organic carbon as the organic matter might have contributed to total acidity through their functional groups like $-\mathrm{COOH}$ and phenolic-OH. Total acidity was present in soil in the $\mathrm{pH}$ range of 5.5 to 7.0 as hydroxyl Al-polymers among acidic soil components whenever soil $\mathrm{pH}$ decreased total acidity increased. 
Table.1 Forms of different type of soil acidity $\left(\operatorname{cmol}(+) \mathrm{kg}^{-1}\right)$ at various soil depths during pre-monsoon as influenced by nitrogen levels

\begin{tabular}{|c|c|c|c|c|c|c|c|c|c|c|c|c|c|c|c|c|}
\hline \multirow{3}{*}{ Soil type } & \multicolumn{16}{|c|}{ Soil depth } \\
\hline & & \multicolumn{5}{|c|}{$0-30 \mathrm{~cm}$} & \multicolumn{5}{|c|}{$30-60 \mathrm{~cm}$} & \multicolumn{4}{|c|}{$60-90 \mathrm{~cm}$} & \multirow[b]{2}{*}{ PDA } \\
\hline & & Ex. A. & $\begin{array}{l}\text { Al. } \\
\text { A. }\end{array}$ & TA & TPA & PDA & Ex. A. & $\begin{array}{l}\text { Al. } \\
\text { A. }\end{array}$ & TA & TPA & PDA & $\begin{array}{l}\text { Ex. } \\
\text { A. }\end{array}$ & $\begin{array}{l}\text { Al. } \\
\text { A. }\end{array}$ & TA & TPA & \\
\hline \multirow[t]{3}{*}{ Inceptisol } & $<92$ & $\begin{array}{l}0.61 \\
(8.9)\end{array}$ & $\begin{array}{c}0.84 \\
(12.3)\end{array}$ & $\begin{array}{c}0.96 \\
(14.0)\end{array}$ & $\begin{array}{c}2.52 \\
(36.8)\end{array}$ & $\begin{array}{c}1.91 \\
(27.9)\end{array}$ & $\begin{array}{l}0.45 \\
(8.4)\end{array}$ & $\begin{array}{c}0.80 \\
(14.9)\end{array}$ & $\begin{array}{c}0.82 \\
(15.3)\end{array}$ & $\begin{array}{c}1.87 \\
(34.9)\end{array}$ & $\begin{array}{c}1.42 \\
(26.5)\end{array}$ & $\begin{array}{l}0.32 \\
(7.7)\end{array}$ & $\begin{array}{c}0.57 \\
(13.7)\end{array}$ & $\begin{array}{c}0.72 \\
(17.3)\end{array}$ & $\begin{array}{c}1.43 \\
(34.4)\end{array}$ & $\begin{array}{c}1.11 \\
(26.74)\end{array}$ \\
\hline & $92-138$ & $0.76(10.1)$ & $\begin{array}{c}0.91 \\
(12.1)\end{array}$ & $\begin{array}{c}1.24 \\
(16.5)\end{array}$ & $\begin{array}{c}2.67 \\
(35.6)\end{array}$ & $\begin{array}{c}1.92 \\
(25.6)\end{array}$ & $\begin{array}{l}0.47 \\
(8.3)\end{array}$ & $\begin{array}{c}0.85 \\
(15.1)\end{array}$ & $\begin{array}{c}0.80 \\
(14.2)\end{array}$ & $\begin{array}{c}1.98 \\
(35.3)\end{array}$ & $\begin{array}{c}1.50 \\
(26.7)\end{array}$ & $\begin{array}{l}0.38 \\
(7.4)\end{array}$ & $\begin{array}{c}0.76 \\
(14.9)\end{array}$ & $\begin{array}{c}0.69 \\
(13.6)\end{array}$ & $\begin{array}{c}1.81 \\
(35.7)\end{array}$ & $\begin{array}{c}1.43 \\
(28.2)\end{array}$ \\
\hline & $>138$ & $\begin{array}{c}0.94 \\
(\mathbf{1 0 . 9})\end{array}$ & $\begin{array}{c}1.08 \\
(12.6)\end{array}$ & $\begin{array}{c}1.36 \\
(15.8)\end{array}$ & $\begin{array}{c}3.06 \\
(\mathbf{3 5 . 7})\end{array}$ & $\begin{array}{c}2.13 \\
(24.8)\end{array}$ & $\begin{array}{l}0.53 \\
\mathbf{( 7 . 8 )}\end{array}$ & $\begin{array}{c}1.07 \\
(15.7)\end{array}$ & $\begin{array}{c}0.91 \\
(13.4)\end{array}$ & $\begin{array}{c}2.40 \\
(\mathbf{3 5 . 3})\end{array}$ & $\begin{array}{c}1.88 \\
(27.7)\end{array}$ & $\begin{array}{l}0.40 \\
(6.6)\end{array}$ & $\begin{array}{c}0.85 \\
(14.0)\end{array}$ & $\begin{array}{c}0.86 \\
(14.2)\end{array}$ & $\begin{array}{c}2.16 \\
\mathbf{( 3 5 . 8 )}\end{array}$ & $\begin{array}{c}1.76 \\
(29.1)\end{array}$ \\
\hline \multirow[t]{3}{*}{ Alfisols } & $<92$ & $\begin{array}{l}0.44 \\
(6.9)\end{array}$ & $\begin{array}{c}0.84 \\
(13.2)\end{array}$ & $\begin{array}{c}0.90 \\
(14.4)\end{array}$ & $\begin{array}{c}2.31 \\
(36.3)\end{array}$ & $\begin{array}{c}1.87 \\
(29.4)\end{array}$ & $\begin{array}{l}0.26 \\
(5.4)\end{array}$ & $\begin{array}{c}0.78 \\
(16.1)\end{array}$ & $\begin{array}{c}0.72 \\
(14.9)\end{array}$ & $\begin{array}{c}1.67 \\
(34.5)\end{array}$ & $\begin{array}{c}1.41 \\
(29.1)\end{array}$ & $\begin{array}{l}0.16 \\
(4.2)\end{array}$ & $\begin{array}{c}0.41 \\
(10.9)\end{array}$ & $\begin{array}{c}0.72 \\
(19.3)\end{array}$ & $\begin{array}{c}1.30 \\
(34.8)\end{array}$ & $\begin{array}{c}1.14 \\
(30.5)\end{array}$ \\
\hline & $92-138$ & $\begin{array}{l}0.57 \\
(8.0)\end{array}$ & $\begin{array}{c}0.90 \\
(12.7)\end{array}$ & $\begin{array}{c}1.4 \\
(14.7)\end{array}$ & $\begin{array}{c}2.55 \\
(36.1)\end{array}$ & $\begin{array}{c}1.99 \\
(28.2)\end{array}$ & $\begin{array}{l}0.42 \\
(6.3)\end{array}$ & $\begin{array}{c}0.69 \\
(10.4)\end{array}$ & $\begin{array}{c}1.12 \\
(16.8)\end{array}$ & $\begin{array}{c}2.41 \\
(36.3)\end{array}$ & $\begin{array}{c}1.99 \\
(30.0)\end{array}$ & $\begin{array}{l}0.30 \\
(5.4)\end{array}$ & $\begin{array}{c}0.59 \\
(10.7)\end{array}$ & $\begin{array}{c}0.88 \\
( \\
15.9)\end{array}$ & $\begin{array}{c}2.02 \\
(36.6)\end{array}$ & $\begin{array}{c}1.72 \\
(31.2)\end{array}$ \\
\hline & $>138$ & $\begin{array}{l}0.71 \\
(\mathbf{8 . 9})\end{array}$ & $\begin{array}{c}1.07 \\
(13.4)\end{array}$ & $\begin{array}{c}1.16 \\
(14.5)\end{array}$ & $\begin{array}{c}2.86 \\
(\mathbf{3 5 . 9})\end{array}$ & $\begin{array}{c}2.16 \\
(27.1)\end{array}$ & $\begin{array}{l}0.45 \\
(6.7)\end{array}$ & $\begin{array}{c}0.87 \\
(13.0)\end{array}$ & $\begin{array}{c}0.98 \\
(14.6)\end{array}$ & $\begin{array}{c}2.42 \\
(\mathbf{3 6 . 2})\end{array}$ & $\begin{array}{c}1.96 \\
(29.3)\end{array}$ & $\begin{array}{l}0.36 \\
(6.4)\end{array}$ & $\begin{array}{c}0.67 \\
(12.0)\end{array}$ & $\begin{array}{c}0.85 \\
(15.3)\end{array}$ & $\begin{array}{c}2.02 \\
(\mathbf{3 6 . 3})\end{array}$ & $\begin{array}{c}1.65 \\
(29.7)\end{array}$ \\
\hline \multirow[t]{3}{*}{ Vertisols } & $<92$ & $\begin{array}{l}0.44 \\
(8.9)\end{array}$ & $\begin{array}{c}1.00 \\
(20.3)\end{array}$ & $\begin{array}{c}0.72 \\
(14.6)\end{array}$ & $\begin{array}{c}1.61 \\
(32.6)\end{array}$ & $\begin{array}{c}1.16 \\
(23.5)\end{array}$ & $\begin{array}{l}0.32 \\
(6.9)\end{array}$ & $\begin{array}{c}0.81 \\
(17.6)\end{array}$ & $\begin{array}{c}0.67 \\
(14.6)\end{array}$ & $\begin{array}{c}1.55 \\
(33.8)\end{array}$ & $\begin{array}{c}1.23 \\
(26.8)\end{array}$ & $\begin{array}{l}0.22 \\
(5.6)\end{array}$ & $\begin{array}{c}0.75 \\
(19.2)\end{array}$ & $\begin{array}{c}0.59 \\
(15.1)\end{array}$ & $\begin{array}{c}1.28 \\
(32.8)\end{array}$ & $\begin{array}{c}1.06 \\
(27.1)\end{array}$ \\
\hline & $92-138$ & $\begin{array}{l}0.52 \\
(7.6)\end{array}$ & $\begin{array}{c}1.00 \\
(14 . \\
7)\end{array}$ & $\begin{array}{c}0.92 \\
(13.5)\end{array}$ & $\begin{array}{c}2.44 \\
(35.8)\end{array}$ & $\begin{array}{c}1.92 \\
(28.2)\end{array}$ & $\begin{array}{l}0.36 \\
(6.8)\end{array}$ & $\begin{array}{c}0.95 \\
(18.1)\end{array}$ & $\begin{array}{c}0.81 \\
(15.4)\end{array}$ & $\begin{array}{c}1.74 \\
(33.2)\end{array}$ & $\begin{array}{c}1.38 \\
(26.3)\end{array}$ & $\begin{array}{l}0.25 \\
(5.5)\end{array}$ & $\begin{array}{c}0.78 \\
(17.4)\end{array}$ & $\begin{array}{c}0.70 \\
(15.6)\end{array}$ & $\begin{array}{c}1.50 \\
(33.4)\end{array}$ & $\begin{array}{c}1.25 \\
(27.9)\end{array}$ \\
\hline & $>138$ & $\begin{array}{l}0.71 \\
(\mathbf{9 . 6 )}\end{array}$ & $\begin{array}{c}1.25 \\
(17.0)\end{array}$ & $\begin{array}{c}1.11 \\
(15.1)\end{array}$ & $\begin{array}{c}2.46 \\
(\mathbf{3 3 . 6})\end{array}$ & $\begin{array}{c}1.79 \\
(24.2)\end{array}$ & $\begin{array}{l}0.48 \\
\mathbf{( 7 . 7 )}\end{array}$ & $\begin{array}{c}1.07 \\
(17.2)\end{array}$ & $\begin{array}{c}0.94 \\
(15.1)\end{array}$ & $\begin{array}{c}2.10 \\
(33.7)\end{array}$ & $\begin{array}{c}1.63 \\
(26.2)\end{array}$ & $\begin{array}{l}0.34 \\
\text { (7.0) }\end{array}$ & $\begin{array}{c}0.72 \\
(14.9)\end{array}$ & $\begin{array}{c}0.73 \\
(15.1)\end{array}$ & $\begin{array}{c}1.66 \\
\mathbf{( 3 4 . 5 )}\end{array}$ & $\begin{array}{c}1.36 \\
(28.2)\end{array}$ \\
\hline
\end{tabular}

0.61 (soil acidity mean) $\quad$ 1. Ex. A = Exchangeable acidity 2. Al. A. $=$ Aluminum acidity 3. TA $=$ Total acidity 4 . TPA $=$ Total potential (8.9) \% contribution acidity 5. $\mathrm{PDA}=\mathrm{pH}$ dependent acidity 
Table.2 Forms of different types of soil acidity $\left(\operatorname{cmol}(+) \mathrm{kg}^{-1}\right)$ at various soil depths during mid-monsoon as influence by nitrogen level

\begin{tabular}{|c|c|c|c|c|c|c|c|c|c|c|c|c|c|c|c|c|}
\hline \multirow{3}{*}{ Soil type } & \multicolumn{16}{|c|}{ Soil depth } \\
\hline & & \multicolumn{5}{|c|}{$0-30 \mathrm{~cm}$} & \multicolumn{5}{|c|}{$30-60 \mathrm{~cm}$} & \multicolumn{4}{|c|}{$60-90 \mathrm{~cm}$} & \multirow[b]{2}{*}{ PDA } \\
\hline & & Ex. A. & Al. A. & TA & TPA & PDA & Ex. A. & Al. A. & TA & TPA & PDA & $\begin{array}{c}\text { Ex. } \\
\text { A. }\end{array}$ & Al. A. & TA & TPA & \\
\hline \multirow[t]{3}{*}{ Inceptisol } & $<92$ & $\begin{array}{l}0.76 \\
(\mathbf{9 . 2})\end{array}$ & $\begin{array}{c}0.98 \\
(11.9)\end{array}$ & $\begin{array}{c}1.40 \\
(17.0)\end{array}$ & $\begin{array}{c}2.91 \\
(35.5)\end{array}$ & $\begin{array}{c}2.14 \\
(26.1)\end{array}$ & $\begin{array}{l}0.46 \\
\mathbf{( 7 . 5 )}\end{array}$ & $\begin{array}{c}0.76 \\
(12.3)\end{array}$ & $\begin{array}{c}0.95 \\
(15.4)\end{array}$ & $\begin{array}{c}2.21 \\
(\mathbf{3 6 . 0})\end{array}$ & $\begin{array}{c}1.75 \\
(28.5)\end{array}$ & $\begin{array}{l}0.29 \\
\mathbf{( 5 . 6 )}\end{array}$ & $\begin{array}{c}0.65 \\
(12.5)\end{array}$ & $\begin{array}{c}0.86 \\
(16.6)\end{array}$ & $\begin{array}{c}1.83 \\
(\mathbf{3 5 . 3})\end{array}$ & $\begin{array}{c}1.54 \\
(29.7)\end{array}$ \\
\hline & $92-138$ & $\begin{array}{c}0.97 \\
(\mathbf{1 1 . 1})\end{array}$ & $\begin{array}{c}1.21 \\
(13.8)\end{array}$ & $\begin{array}{c}1.58 \\
(18.0)\end{array}$ & $\begin{array}{c}2.97 \\
(\mathbf{3 4 . 0})\end{array}$ & $\begin{array}{c}2.00 \\
(22.9)\end{array}$ & $\begin{array}{l}0.66 \\
(9.7)\end{array}$ & $\begin{array}{c}1.07 \\
(15.7)\end{array}$ & $\begin{array}{c}0.99 \\
(14.6)\end{array}$ & $\begin{array}{c}2.36 \\
(\mathbf{3 4 . 8})\end{array}$ & $\begin{array}{c}1.70 \\
(25.0)\end{array}$ & $\begin{array}{l}0.46 \\
\mathbf{( 7 . 7 )}\end{array}$ & $\begin{array}{c}0.98 \\
(17.2)\end{array}$ & $\begin{array}{c}0.85 \\
(14.9)\end{array}$ & $\begin{array}{c}1.93 \\
\mathbf{( 3 4 . 0 )}\end{array}$ & $\begin{array}{c}1.47 \\
(25.8)\end{array}$ \\
\hline & $>138$ & $\begin{array}{c}1.31 \\
(14.0)\end{array}$ & $\begin{array}{c}1.11 \\
(11.9)\end{array}$ & $\begin{array}{c}1.63 \\
(17.5)\end{array}$ & $\begin{array}{c}3.28 \\
(35.2)\end{array}$ & $\begin{array}{c}1.97 \\
(21.1)\end{array}$ & $\begin{array}{c}0.77 \\
(10.6)\end{array}$ & $\begin{array}{c}1.00 \\
(13.7)\end{array}$ & $\begin{array}{c}1.19 \\
(16.3)\end{array}$ & $\begin{array}{c}2.53 \\
(34.8)\end{array}$ & $\begin{array}{c}1.77 \\
(16.1)\end{array}$ & $\begin{array}{l}0.57 \\
(8.9)\end{array}$ & $\begin{array}{c}0.89 \\
(13.9)\end{array}$ & $\begin{array}{c}0.95 \\
(14.8)\end{array}$ & $\begin{array}{c}2.27 \\
(35.5)\end{array}$ & $\begin{array}{c}1.70 \\
(26.6)\end{array}$ \\
\hline \multirow[t]{3}{*}{ Alfisols } & $<92$ & $\begin{array}{l}0.68 \\
(8.5)\end{array}$ & $\begin{array}{c}1.14 \\
(14.3)\end{array}$ & $\begin{array}{c}1.14 \\
(14.3)\end{array}$ & $\begin{array}{c}2.84 \\
(\mathbf{3 5 . 6})\end{array}$ & $\begin{array}{c}2.16 \\
(27.1)\end{array}$ & $\begin{array}{l}0.39 \\
(\mathbf{6 . 5})\end{array}$ & $\begin{array}{c}0.95 \\
(15.8)\end{array}$ & $\begin{array}{c}0.91 \\
(15.1)\end{array}$ & $\begin{array}{c}2.06 \\
\mathbf{( 3 4 . 3 )}\end{array}$ & $\begin{array}{c}1.68 \\
(28.0)\end{array}$ & $\begin{array}{l}0.24 \\
\mathbf{( 4 . 8 )}\end{array}$ & $\begin{array}{c}0.74 \\
(14.8)\end{array}$ & $\begin{array}{c}0.79 \\
(15.9)\end{array}$ & $\begin{array}{c}1.72 \\
\mathbf{( 3 4 . 6 )}\end{array}$ & $\begin{array}{c}1.48 \\
(29.7)\end{array}$ \\
\hline & 92-138 & $\begin{array}{c}0.89 \\
(\mathbf{1 1 . 0})\end{array}$ & $\begin{array}{c}0.98 \\
(12.1)\end{array}$ & $\begin{array}{c}1.27 \\
(15.7)\end{array}$ & $\begin{array}{c}2.87 \\
(\mathbf{3 5 . 6})\end{array}$ & $\begin{array}{c}2.03 \\
(25.2)\end{array}$ & $\begin{array}{l}0.47 \\
\mathbf{( 7 . 2 )}\end{array}$ & $\begin{array}{c}0.87 \\
(13.3)\end{array}$ & $\begin{array}{c}0.99 \\
(15.2)\end{array}$ & $\begin{array}{c}2.32 \\
(\mathbf{3 5 . 6})\end{array}$ & $\begin{array}{c}1.85 \\
(28.4)\end{array}$ & $\begin{array}{l}0.36 \\
(6.5)\end{array}$ & $\begin{array}{c}0.67 \\
(17.7)\end{array}$ & $\begin{array}{c}0.78 \\
(14.2)\end{array}$ & $\begin{array}{c}2.01 \\
(\mathbf{3 6 . 6})\end{array}$ & $\begin{array}{c}1.66 \\
(30.2)\end{array}$ \\
\hline & $>138$ & $\begin{array}{c}0.96 \\
(11.0)\end{array}$ & $\begin{array}{c}1.31 \\
(15.1)\end{array}$ & $\begin{array}{c}1.17 \\
(13.5)\end{array}$ & $\begin{array}{c}3.09 \\
(35.6)\end{array}$ & $\begin{array}{c}2.13 \\
(24.5)\end{array}$ & $\begin{array}{l}0.50 \\
(7.1)\end{array}$ & $\begin{array}{c}1.15 \\
(16.3)\end{array}$ & $\begin{array}{c}0.99 \\
(14.1)\end{array}$ & $\begin{array}{c}2.44 \\
(34.7)\end{array}$ & $\begin{array}{c}1.94 \\
(27.6)\end{array}$ & $\begin{array}{l}0.35 \\
(5.7)\end{array}$ & $\begin{array}{c}0.97 \\
(15.8)\end{array}$ & $\begin{array}{c}0.86 \\
(14.0)\end{array}$ & $\begin{array}{c}2.14 \\
(35.0)\end{array}$ & $\begin{array}{c}1.79 \\
(29.3)\end{array}$ \\
\hline \multirow[t]{3}{*}{ Vertisols } & $<92$ & $\begin{array}{l}0.61 \\
(\mathbf{9 . 2})\end{array}$ & $\begin{array}{c}1.25 \\
(18.8)\end{array}$ & $\begin{array}{c}1.09 \\
(16.4)\end{array}$ & $\begin{array}{c}2.14 \\
\mathbf{( 3 2 . 3 )}\end{array}$ & $\begin{array}{c}1.53 \\
(23.1)\end{array}$ & $\begin{array}{l}0.42 \\
\mathbf{( 7 . 5 )}\end{array}$ & $\begin{array}{c}1.00 \\
(17.9)\end{array}$ & $\begin{array}{c}0.88 \\
(15.7)\end{array}$ & $\begin{array}{c}1.85 \\
\mathbf{( 3 3 . 1 )}\end{array}$ & $\begin{array}{c}1.43 \\
(25.6)\end{array}$ & $\begin{array}{l}0.27 \\
\mathbf{( 5 . 5 )}\end{array}$ & $\begin{array}{c}0.84 \\
(17.1)\end{array}$ & $\begin{array}{c}0.80 \\
(16.3)\end{array}$ & $\begin{array}{c}1.63 \\
\mathbf{( 3 3 . 0 )}\end{array}$ & $\begin{array}{c}1.36 \\
(27.7)\end{array}$ \\
\hline & 92-138 & $\begin{array}{c}0.80 \\
(\mathbf{1 0 . 7})\end{array}$ & $\begin{array}{c}1.04 \\
(14.0)\end{array}$ & $\begin{array}{c}1.27 \\
(17.0)\end{array}$ & $\begin{array}{c}2.56 \\
\mathbf{( 3 4 . 4 )}\end{array}$ & $\begin{array}{c}1.76 \\
(23.6)\end{array}$ & $\begin{array}{l}0.47 \\
\mathbf{( 8 . 3 )}\end{array}$ & $\begin{array}{c}0.81 \\
(14.4)\end{array}$ & $\begin{array}{c}0.85 \\
(15.1)\end{array}$ & $\begin{array}{c}1.98 \\
\mathbf{( 3 5 . 2 )}\end{array}$ & $\begin{array}{c}1.51 \\
(26.8)\end{array}$ & $\begin{array}{l}0.37 \\
\mathbf{( 7 . 5 )}\end{array}$ & $\begin{array}{c}0.63 \\
(12.7)\end{array}$ & $\begin{array}{c}0.78 \\
(15.8)\end{array}$ & $\begin{array}{c}1.76 \\
\mathbf{( 3 5 . 6 )}\end{array}$ & $\begin{array}{c}1.39 \\
(28.1)\end{array}$ \\
\hline & $>138$ & $\begin{array}{c}0.85 \\
(10.0)\end{array}$ & $\begin{array}{c}1.44 \\
(16.9)\end{array}$ & $\begin{array}{c}1.31 \\
(15.4)\end{array}$ & $\begin{array}{c}2.87 \\
(33.8)\end{array}$ & $\begin{array}{c}2.02 \\
(23.7)\end{array}$ & $\begin{array}{l}0.45 \\
(6.6)\end{array}$ & $\begin{array}{c}1.14 \\
(16.8)\end{array}$ & $\begin{array}{c}0.97 \\
(14.3))\end{array}$ & $\begin{array}{c}2.32 \\
(34.3)\end{array}$ & $\begin{array}{c}1.87 \\
(27.7)\end{array}$ & $\begin{array}{l}0.26 \\
(4.2)\end{array}$ & $\begin{array}{c}0.99 \\
(16.1)\end{array}$ & $\begin{array}{c}0.93 \\
(15.1)\end{array}$ & $\begin{array}{c}2.12 \\
(34.6)\end{array}$ & $\begin{array}{c}1.85 \\
(30.2)\end{array}$ \\
\hline \multicolumn{3}{|c|}{$\begin{array}{l}\text { 0.61 (soil acidity mean) } \\
(8.9) \% \text { contribution }\end{array}$} & & & & & & & Geptie & $\begin{array}{l}\mathrm{A}=\mathrm{T} \\
\text { cidity }\end{array}$ & & & & & & \\
\hline
\end{tabular}


Table .3 Forms of different types of soil acidity $\left(\mathrm{cmol}(+) \mathrm{kg}^{-1}\right)$ at various soil depths during Post-monsoon as influence by N-level

\begin{tabular}{|c|c|c|c|c|c|c|c|c|c|c|c|c|c|c|c|c|}
\hline \multirow{3}{*}{ Soil type } & \multicolumn{16}{|c|}{ Soil depth } \\
\hline & & \multicolumn{5}{|c|}{$0-30 \mathrm{~cm}$} & \multicolumn{5}{|c|}{$30-60 \mathrm{~cm}$} & \multicolumn{4}{|c|}{$60-90 \mathrm{~cm}$} & \multirow[b]{2}{*}{ PDA } \\
\hline & & Ex. A. & Al. A. & TA & TPA & PDA & Ex. A. & Al. A. & TA & TPA & PDA & $\begin{array}{l}\text { Ex. } \\
\text { A. }\end{array}$ & Al. A. & $\mathrm{TA}$ & TPA & \\
\hline \multirow[t]{3}{*}{ Inceptisol } & $<92$ & $\begin{array}{l}0.65 \\
(\mathbf{9 . 0})\end{array}$ & $\begin{array}{c}0.92 \\
(12.7)\end{array}$ & $\begin{array}{c}1.17 \\
(16.2)\end{array}$ & $\begin{array}{c}2.56 \\
\mathbf{( 3 5 . 5 )}\end{array}$ & $\begin{array}{c}1.91 \\
(26.4)\end{array}$ & $\begin{array}{l}0.40 \\
\mathbf{( 7 . 0 )}\end{array}$ & $\begin{array}{c}0.71 \\
(12.5)\end{array}$ & $\begin{array}{c}0.86 \\
(15.2)\end{array}$ & $\begin{array}{c}2.04 \\
(\mathbf{3 6 . 1})\end{array}$ & $\begin{array}{c}1.64 \\
(29.0)\end{array}$ & $\begin{array}{l}0.36 \\
\mathbf{( 7 . 9 )}\end{array}$ & $\begin{array}{c}0.66 \\
(14.5)\end{array}$ & $\begin{array}{c}0.76 \\
(16.7)\end{array}$ & $\begin{array}{c}1.56 \\
(\mathbf{3 4 . 3})\end{array}$ & $\begin{array}{c}1.20 \\
(26.4)\end{array}$ \\
\hline & $92-138$ & $\begin{array}{c}0.86 \\
(11.4)\end{array}$ & $\begin{array}{c}0.77 \\
(10.2)\end{array}$ & $\begin{array}{c}1.08 \\
(14.3)\end{array}$ & $\begin{array}{c}2.83 \\
(37.6)\end{array}$ & $\begin{array}{c}1.97 \\
(26.2)\end{array}$ & $\begin{array}{l}0.46 \\
(7.7)\end{array}$ & $\begin{array}{c}0.75 \\
(12.5)\end{array}$ & $\begin{array}{c}0.90 \\
(15.1)\end{array}$ & $\begin{array}{c}2.16 \\
(36.2)\end{array}$ & $\begin{array}{c}1.69 \\
(28.3)\end{array}$ & $\begin{array}{l}0.42 \\
(8.1)\end{array}$ & $\begin{array}{c}0.64 \\
(12.4)\end{array}$ & $\begin{array}{c}0.82 \\
(15.8)\end{array}$ & $\begin{array}{c}1.85 \\
(35.8)\end{array}$ & $\begin{array}{c}1.43 \\
(27.7)\end{array}$ \\
\hline & $>138$ & $\begin{array}{c}1.03 \\
(11.6)\end{array}$ & $\begin{array}{c}0.98 \\
(11.0)\end{array}$ & $\begin{array}{c}1.43 \\
(16.1)\end{array}$ & $\begin{array}{c}3.22 \\
(36.3)\end{array}$ & $\begin{array}{c}2.19 \\
(24.7)\end{array}$ & $\begin{array}{l}0.67 \\
(9.7)\end{array}$ & $\begin{array}{c}0.89 \\
(13.0)\end{array}$ & $\begin{array}{c}1.00 \\
(15.1)\end{array}$ & $\begin{array}{c}2.48 \\
(36.2)\end{array}$ & $\begin{array}{c}1.81 \\
(26.4)\end{array}$ & $\begin{array}{l}0.47 \\
(7.8)\end{array}$ & $\begin{array}{c}0.76 \\
(12.6)\end{array}$ & $\begin{array}{c}0.89 \\
(14.8)\end{array}$ & $\begin{array}{c}2.18 \\
(36.3)\end{array}$ & $\begin{array}{c}1.70 \\
(28.3)\end{array}$ \\
\hline \multirow[t]{3}{*}{ Alfisols } & $<92$ & $\begin{array}{l}0.61 \\
(9.1)\end{array}$ & $\begin{array}{c}0.88 \\
(13.1)\end{array}$ & $\begin{array}{c}1.00 \\
(14.9)\end{array}$ & $\begin{array}{c}2.41 \\
\mathbf{( 3 5 . 9 )}\end{array}$ & $\begin{array}{c}1.80 \\
(26.8)\end{array}$ & $\begin{array}{l}0.40 \\
\mathbf{( 7 . 7 )}\end{array}$ & $\begin{array}{c}0.74 \\
(14.3)\end{array}$ & $\begin{array}{c}0.81 \\
(15.7)\end{array}$ & $\begin{array}{c}1.80 \\
\mathbf{( 3 4 . 9 )}\end{array}$ & $\begin{array}{c}1.40 \\
(27.1)\end{array}$ & $\begin{array}{l}0.32 \\
\mathbf{( 7 . 4 )}\end{array}$ & $\begin{array}{c}0.50 \\
(11.6)\end{array}$ & $\begin{array}{c}0.73 \\
(17.0)\end{array}$ & $\begin{array}{c}1.53 \\
(\mathbf{3 5 . 6})\end{array}$ & $\begin{array}{c}1.21 \\
(28.2)\end{array}$ \\
\hline & $92-138$ & $\begin{array}{c}0.78 \\
(10.5)\end{array}$ & $\begin{array}{c}0.89 \\
(12.0)\end{array}$ & $\begin{array}{c}1.05 \\
(14.1)\end{array}$ & $\begin{array}{c}2.73 \\
(36.8)\end{array}$ & $\begin{array}{c}1.95 \\
(26.3)\end{array}$ & $\begin{array}{c}0.51 \\
(7.5)\end{array}$ & $\begin{array}{c}0.82 \\
(12.1)\end{array}$ & $\begin{array}{c}1.01 \\
(14.9)\end{array}$ & $\begin{array}{c}2.46 \\
(36.3)\end{array}$ & $\begin{array}{c}1.96 \\
(29.0)\end{array}$ & $\begin{array}{l}0.33 \\
(6.3)\end{array}$ & $\begin{array}{c}0.62 \\
(11.9)\end{array}$ & $\begin{array}{c}0.83 \\
(15.9)\end{array}$ & $\begin{array}{c}1.88 \\
(36.0)\end{array}$ & $\begin{array}{c}1.55 \\
(29.7)\end{array}$ \\
\hline & $>138$ & $\begin{array}{c}0.87 \\
(11.0)\end{array}$ & $\begin{array}{c}1.02 \\
(12.9)\end{array}$ & $\begin{array}{c}1.07 \\
(13.6)\end{array}$ & $\begin{array}{c}2.89 \\
(36.7)\end{array}$ & $\begin{array}{c}2.02 \\
(25.7)\end{array}$ & $\begin{array}{l}0.41 \\
(6.4)\end{array}$ & $\begin{array}{c}0.75 \\
(11.7)\end{array}$ & $\begin{array}{c}0.84 \\
(13.1)\end{array}$ & $\begin{array}{c}2.40 \\
(37.5)\end{array}$ & $\begin{array}{c}1.99 \\
(31.1)\end{array}$ & $\begin{array}{c}0.37 \\
(7.0)\end{array}$ & $\begin{array}{c}0.55 \\
(10.4)\end{array}$ & $\begin{array}{c}0.76 \\
(14.4)\end{array}$ & $\begin{array}{c}1.97 \\
(37.5)\end{array}$ & $\begin{array}{c}1.60 \\
(30.4)\end{array}$ \\
\hline \multirow[t]{3}{*}{ Vertisols } & $<92$ & $\begin{array}{l}0.46 \\
\mathbf{( 7 . 7 )}\end{array}$ & $\begin{array}{c}0.92 \\
(15.5)\end{array}$ & $\begin{array}{c}0.77 \\
(13.0)\end{array}$ & $\begin{array}{c}2.12 \\
(\mathbf{3 5 . 8})\end{array}$ & $\begin{array}{c}1.65 \\
(27.8)\end{array}$ & $\begin{array}{l}0.37 \\
(\mathbf{8 . 1})\end{array}$ & $\begin{array}{c}0.80 \\
(17.5)\end{array}$ & $\begin{array}{c}0.64 \\
(14.0)\end{array}$ & $\begin{array}{c}1.56 \\
(\mathbf{3 4 . 2})\end{array}$ & $\begin{array}{c}1.19 \\
(26.0)\end{array}$ & $\begin{array}{l}0.26 \\
(\mathbf{6 . 4})\end{array}$ & $\begin{array}{c}0.55 \\
(13.6)\end{array}$ & $\begin{array}{c}0.59 \\
(14.6)\end{array}$ & $\begin{array}{c}1.44 \\
\mathbf{( 3 5 . 8 )}\end{array}$ & $\begin{array}{c}1.18 \\
(29.3)\end{array}$ \\
\hline & $92-138$ & $\begin{array}{l}0.67 \\
(9.5)\end{array}$ & $\begin{array}{c}0.92 \\
(13.1)\end{array}$ & $\begin{array}{c}1.09 \\
(15.5)\end{array}$ & $\begin{array}{c}2.49 \\
(35.6)\end{array}$ & $\begin{array}{c}1.82 \\
(26.0)\end{array}$ & $\begin{array}{l}0.36 \\
(6.8)\end{array}$ & $\begin{array}{c}0.82 \\
(15.5)\end{array}$ & $\begin{array}{c}0.83 \\
(15.7)\end{array}$ & $\begin{array}{c}1.81 \\
(34.3)\end{array}$ & $\begin{array}{c}1.45 \\
(27.5)\end{array}$ & $\begin{array}{l}0.27 \\
(5.5)\end{array}$ & $\begin{array}{c}0.72 \\
(14 \\
.8)\end{array}$ & $\begin{array}{c}0.72 \\
(14.8)\end{array}$ & $\begin{array}{c}1.70 \\
(35.1)\end{array}$ & $\begin{array}{c}1.43 \\
(29.5)\end{array}$ \\
\hline & $>138$ & $\begin{array}{l}0.69 \\
(8.9)\end{array}$ & $\begin{array}{c}1.14 \\
(14.7)\end{array}$ & $\begin{array}{c}1.16 \\
(15.0)\end{array}$ & $\begin{array}{c}2.71 \\
(35.1)\end{array}$ & $\begin{array}{c}2.01 \\
(26.0)\end{array}$ & $\begin{array}{l}0.45 \\
(7.4)\end{array}$ & $\begin{array}{c}0.91 \\
(15.0)\end{array}$ & $\begin{array}{c}0.96 \\
(15.8)\end{array}$ & $\begin{array}{c}2.10 \\
(34.5)\end{array}$ & $\begin{array}{c}1.65 \\
(27.1)\end{array}$ & $\begin{array}{l}0.26 \\
(5.3)\end{array}$ & $\begin{array}{c}0.64 \\
(13.2)\end{array}$ & $\begin{array}{c}0.62 \\
(12.8)\end{array}$ & $\begin{array}{c}1.78 \\
(37.0)\end{array}$ & $\begin{array}{c}1.52 \\
(31.5)\end{array}$ \\
\hline \multicolumn{3}{|c|}{$\begin{array}{l}\text { 0.61 (soil acidity mean) } \\
\text { (8.9) \% contribution }\end{array}$} & \multicolumn{14}{|c|}{$\begin{array}{c}\text { 1. Ex. A }=\text { Exchangeable acidity } 2 . \text { Al } . \mathrm{A} .=\text { Aluminum acidity } 3 . \mathrm{TA}=\text { Total acidity } 4 . \mathrm{TPA}=\text { Total potential acidity } 5 . \mathrm{PDA}=\mathrm{pH} \\
\text { dependent acidity }\end{array}$} \\
\hline
\end{tabular}


However, surface soils recorded higher total acidity content compared to subsurface layer. Total acidity was highest in Inceptisol followed by Alfisol than Vertisol. simillar results were also reported by Bhat et.al (2017), Bandyopadhyay and Chattopadhyay (1997), Ananthnarayana and Ravi (1997). and Pati and Mukhopadhyay (2010).

\section{Acknowledgements}

We are very grateful to IGKV for providing the laboratory without which the study would not have been possible. We would also like to acknowledge the kind cooperation extended by the staff of Soil Science and Agricultural Chemistry Raipur in completing this research projects

\section{References}

Ananthanarayanaya, R. and Ravi, M. V., 1997. Nature of soil acidity of coffee growing soils of Karnataka.J. Indian Soc. Soil Sci., 45(2): 384-385.

Bandyopadhyay, P. K. and Chattopadhyay, G. N. 1997. Nature of acidity in some Alfisols and Inceptisols of Birbhum district of West Bengal. J. Indian Soc. Soil Sc., 45: 5-8.

Baruah, T. C. and Barthakur, H. P. 1999. A Text book of soil analysis..Vikash Publishing House Pvt. Ltd., New Delhi, 94-103.

Bhat, J.A., Kundu, M.C., Mandal, B. and Hazra, G. C. 2017. Nature of acidity in Alfisols, Entisols and Inceptisols in relation to soil properties.
Communications in Soil Science and Plant Analysis, 48(4)395-404

Das, A. N., Laskar, B. K., De, G. K. and Dehnath, N. C. 1991. Nature of acidity of some acid soils of West Bengal.Journal of the Indian Society of Soil Science, 39: 246 -251.

Kappen, G. 1934. Pochvennaya, Kislotmost,Selkhogiz, Moscow. cf. Journal Indian Socity Soils Science, 39: 246.

Kumar, K. 1995. Nature of acidity and its relation with lime requirement of some acid soils of Manipur hills. J. Hill Res., 10(2): 131-135.

Pati, R. and Mukhopadhyay, D. 2010. Forms of soil acidity and the distribution of DTPA-extractable micronutrients in some soils of West Bengal.19th World Congress of Soil Science, Soil Solutions for a Changing World, 14-16.

Peech, M., Cowan, R. L. and Baker, J. H. 1962. A critical study of the barium chloride-tri ethanolamine and ammonium acetate methods for determining the exchangeable hydrogen content of soils.Proceedings of Soil Science Society of America, 26: 37 - 40.

Reza, S. K., Baruah, U., Bandyopadhyay, S., Sarkar, D. and Dutta D. P. 2012. Characterization of soil acidity under different land uses in Assam.Agropedology, 22(2): 123-127.

Sharma, D. and Sarangthem, I. 2017. Nature of acidity and lime requirement in 'acid soils of Manipur. International Journal of Advance Scientific Research and Engineering Trends, 2 (1):51-58.

\section{How to cite this article:}

Hareesh Kumar, S. S. Sengar, Bharat Desmukh and Singh, R. N. 2020. Different Types of Soil Acidity as Influenced by Nitrogen Application and Soil Types in Dhamtari Block of Chhattisgarh, India. Int.J.Curr.Microbiol.App.Sci. 9(01): 152-159. doi: https://doi.org/10.20546/ijcmas.2020.901.017 